\title{
Peritoneal myofibroblasts at metastatic foci promote dissemination of pancreatic cancer
}

\author{
SHIN AKAGAWA ${ }^{1}$, KENOKI OHUCHIDA ${ }^{1,3}$, NOBUHIRO TORATA ${ }^{1}$, MASAMI HATTORI $^{2}$, DAIKI EGUCHI $^{1}$, \\ KENJI FUJIWARA ${ }^{1}$, SHINGO KOZONO $^{1}$, LIN CUI $^{1}$, NAOKI IKENAGA $^{1}$, TAKAO OHTSUKA $^{1}$, \\ SHINICHI AISHIMA ${ }^{2}$, KAZUHIRO MIZUMOTO ${ }^{1,4}$, YOSHINAO ODA $^{2}$ and MASAO TANAKA ${ }^{1}$ \\ Departments of ${ }^{1}$ Surgery and Oncology, ${ }^{2}$ Anatomic Pathology and ${ }^{3}$ Advanced Medical Initiatives, Graduate School \\ of Medical Sciences, Kyushu University; ${ }^{4}$ Kyushu University Hospital Cancer Center, Fukuoka, Japan
}

Received November 30, 2013; Accepted December 27, 2013

DOI: 10.3892/ijo.2014.2391

\begin{abstract}
Myofibroblasts in the stroma of pancreatic cancers promote tumor proliferation, invasion and metastasis by increasing extracellular matrix and secretion of several growth factors. In contrast, the role of myofibroblasts at peritoneally disseminated sites of pancreatic cancer has not yet been determined. This study was designed to assess the role of myofibroblasts at peritoneally disseminated sites of pancreatic cancer. Three primary cultures of human peritoneal myofibroblasts (hPMFs) were established from disseminated sites of pancreatic cancer and their interactions with the SUIT-2 and CAPAN-1 human pancreatic cancer cell lines were analyzed in vitro. Using a model in $\mathrm{BALB} / \mathrm{c} \mathrm{nu} / \mathrm{nu}$ mice, we compared the dissemination ability of intraperitoneally implanted pancreatic cancer cells, with and without hPMFs, and examined the presence of green fluorescent protein (GFP)-labeled hPMFs at peritoneally disseminated sites in mice. hPMFs significantly promoted the migration and invasion of pancreatic cancer cells $(\mathrm{P}<0.05)$, while the cancer cells significantly promoted the migration and invasion of hPMFs $(\mathrm{P}<0.05)$. In vivo, the number of peritoneally disseminated nodules, more than $3 \mathrm{~mm}$ in size, was significantly greater in mice implanted with cancer cells plus hPMFs compared to mice implanted with cancer cells alone, with GFP-labeled hPMFs surviving in the peritoneal cavity of the former. hPMFs promote the peritoneal dissemination of pancreatic cancer. The cancer-stromal cell interaction in the peritoneal cavity may be a new therapeutic target to prevent the dissemination of pancreatic cancer.
\end{abstract}

Correspondence to: Dr Kenoki Ohuchida or Dr Kazuhiro Mizumoto, Department of Surgery and Oncology, Graduate School of Medical Sciences, Kyushu University, 3-1-1 Maidashi, Fukuoka 812-8582, Japan

E-mail: kenoki@med.kyushu-u.ac.jp

E-mail: mizumoto@med.kyushu-u.ac.jp

Keywords: pancreatic cancer, myofibroblast, peritoneal dissemination, cancer-stromal interaction

\section{Introduction}

Pancreatic cancer is one of the most lethal cancers, with a 5-year overall survival (OS) rate of only $4 \%$ (1). Despite developments in diagnostic imaging, most patients with pancreatic cancer are diagnosed with an advanced stage of the disease. For example, almost 33\% of 100,313 pancreatic cancer patients diagnosed from 1989 through 1995 had metastasis (2). Moreover, most patients who undergo curative resection develop incurable local relapses, liver metastases and/or peritoneal dissemination.

A prospective study of 100 patients with peritoneal carcinomatosis resulting from non-gynecological malignancies found that the second most common primary tumor was pancreatic cancer (20\%) (3). A French multicentric prospective study of 370 peritoneal carcinomatosis patients with non-gynecological malignancies showed a median OS of 3.1 months, but was only 2.1 months in those with pancreatic cancer (4). These data suggest that the novel therapies to control the peritoneal dissemination of pancreatic cancer may improve patient survival.

Pancreatic cancer is one of the most stroma-rich cancers and characterized by excessive desmoplasia, which plays a crucial role in its aggressive behavior $(5,6)$. The stroma in these tumors is very heterogeneous, consisting of cellular and acellular components, including fibroblasts, myofibroblasts, immune cells, blood vessels, extracellular matrix (ECM) and soluble proteins such as cytokines and growth factors (7). Stromal myofibroblasts derived from the primary site of pancreatic cancer were shown to enhance the progression of pancreatic cancer (8). Moreover, stromal components were shown to promote the malignant behavior of pancreatic cancer cells, with myofibroblasts being the especially associated with cancer-stromal cell interactions in primary tumors (7,9-12). Few studies, however, assessed cancer-stromal cell interactions at peritoneally disseminated sites (13-15).

Myofibroblasts are regarded as playing a central role in pathogenesis of peritoneal fibrosis, which provide a favorable environment for the dissemination of cancer cells (13). Although myofibroblasts have been reported to derive from resident peritoneal fibroblasts, human peritoneal mesothelial cells (hPMCs) $(16,17)$, bone marrow progenitor cells or the primary tumor itself $(18,19)$, the origin of these myofibroblasts has not been clearly established. Also, during the initial stages of peritoneal 
metastasis, cancer cells have been reported to attach to areas of exposure of collagen-rich connective tissue matrices because of the lack of mesothelium (20-23). Thus, the interactions between cancer cells derived from the primary tumor and submesothelium layer components such as myofibroblasts may be a key to peritoneal dissemination (24). Hepatocyte growth factors (HGF) produced by human peritoneal myofibroblasts have been found to promote the peritoneal dissemination of gastric cancer (25), and myofibroblasts in omentum were shown to be activated by tumor cells and to promote the growth, adhesion and invasiveness of ovarian cancer (14). However, the roles of peritoneal myofibroblasts and their matrices in the peritoneal dissemination of pancreatic cancer remain unclear. The development of novel therapies to control the peritoneal dissemination of pancreatic cancer requires further understanding of the mechanisms of induction and the roles of peritoneal myofibroblasts in the process of dissemination.

In the present study, we established three primary cultures of human peritoneal myofibroblasts (hPMFs) isolated from peritoneally disseminated nodules of pancreatic cancer and investigated the interactions between primary cultures of hPMFs and pancreatic cancer cells in vitro and in vivo.

\section{Materials and methods}

Tissues, cells and culture conditions. Peritoneally disseminated tissues were obtained from 3 patients who underwent palliative (bypass) operations for unresectable pancreatic cancer at our institution under an Institutional Review Board-approved protocol following informed consent. Human peritoneal myofibroblasts (hPMFs) were isolated from these fresh surgical specimens using the outgrowth method in our laboratory $(26,27)$, and the identity of these hPMFs was confirmed by morphology (spindle-shaped cells) and immunofluorescence staining for $\alpha$-SMA, vimentin and cytokeratin 18 (CK18) $(8,28)$. Cells at passage numbers 2-4 were used for all assays. Two pancreatic cancer cell lines, SUIT-2 (Japan Health Sciences Foundation) and CAPAN-1 (American Type Culture Collection, Manassas, VA, USA), were maintained as previously described (29).

Immunohistochemistry and histopathology. hPMFs isolated from peritoneally disseminated sites of pancreatic cancer were evaluated by $\mathrm{H} \& \mathrm{E}$ and $\alpha$-SMA immunohistochemical staining. Immunohistochemical staining was performed using a Histofine SAB-PO kit (Nichirei, Tokyo, Japan). Tissues were sectioned to a thickness of $4 \mu \mathrm{m}$ and were incubated with antibody overnight at $4^{\circ} \mathrm{C}$. Human tissues were incubated with mouse monoclonal anti- $\alpha$-SMA antibody (1:50; Dako, Glostrup, Denmark) and mouse tissues were incubated with rabbit polyclonal anti- $\alpha$-SMA antibody (1:50; Abcam, Cambridge, MA, USA) (30). Cells were considered positively stained when either the membrane or cytoplasm was stained. All slides were evaluated independently by two investigators blinded to knowledge of the clinical features of each patient.

Immunofluorescence staining. hPMFs were incubated with by rabbit polyclonal anti-vimentin antibody (1:100; Abcam), mouse monoclonal anti-CK18 antibody (1:100; sc-6259, Santa Cruz Biotechnology, Santa Cruz, CA, USA) and anti- $\alpha-$ SMA antibody (1:50; Dako). For comparison, human mesothelial cells from cancerous ascites of pancreatic cancer were incubated with antibodies to vimentin and CK18.

Matrigel invasion and migration assays (indirect co-culture). Cell invasion was measured by counting the number of cells that invaded Matrigel-coated Transwell chambers with $8-\mu \mathrm{m}$ pores (BD Biosciences, Franklin Lakes, NJ, USA), as previously described (31). Briefly, Transwell inserts were coated with $20 \mu \mathrm{g} /$ well Matrigel (BD Biosciences). Each lower well of a 24-well plate was seeded with myofibroblasts $\left(2.0 \times 10^{4} /\right.$ well $)$ in $750 \mu \mathrm{l}$ of DMEM supplemented with $10 \% \mathrm{FBS}$ or medium alone and incubated for $24 \mathrm{~h}$. Cancer cells $\left(4.0 \times 10^{4} /\right.$ well) in $250 \mu \mathrm{l}$ of DMEM supplemented with $10 \%$ FBS were seeded into each upper well. After $69 \mathrm{~h}$ of incubation, cells on the lower surface of the Matrigel-coated membrane were fixed with $70 \%$ ethanol, stained with $\mathrm{H} \& \mathrm{E}$, and counted in five randomly selected fields at a x100, magnification under a light microscope. The mobility of SUIT-2 and CAPAN-1 pancreatic cancer cells was assessed using uncoated Transwell inserts and incubation times of 24 and $48 \mathrm{~h}$, respectively. To assess the invasiveness of myofibroblasts, each lower well was seeded with cancer cells $\left(4.0 \times 10^{4} /\right.$ well) in $750 \mu \mathrm{l}$ of DMEM supplemented with $10 \%$ FBS or medium alone and incubated for $24 \mathrm{~h}$. Myofibroblasts $\left(1.5 \times 10^{4} /\right.$ well $)$ in $250 \mu \mathrm{l}$ of DMEM supplemented with $10 \%$ FBS were seeded in each upper well and incubated for $20 \mathrm{~h}$ for the invasion assay and $14 \mathrm{~h}$ for the migration assay. The results were expressed as the mean number of invaded cells per field. Each experiment was carried out in triplicate wells and repeated at least 3 times.

Animal models. All experiments with mice were conducted with the approval of the Ethics Committee of Kyushu University. The model of intraperitoneal implantation of BALB/c nu/nu mice (5-6 weeks of age, Kyudo Co.) was used to analyze the dissemination activity of cancer cells alone and cancer cells plus hPMFs. All animals were bred in laminar-flow cabinets under specific pathogen-free conditions. Prior to implanting, the cells were briefly treated with trypsin/EDTA and washed twice with serum-free medium. The mice were anesthetized with ether and suspensions of $2 \times 10^{5}$ SUIT- 2 or $1 \times 10^{6}$ CAPAN-1 cells in $200 \mu \mathrm{l}$ PBS with or without $1 \times 10^{6}$ hPMFs in $200 \mu 1$ PBS were transplanted into the peritoneal cavity of groups of 10 mice. All mice were sacrificed 28 days later, and disseminated nodules $>3 \mathrm{~mm}$ in size were counted. Each experiment was repeated 3 times.

Green fluorescent protein (GFP)-labeled myofibroblasts in vivo. The GIPZ non-silencing control vector for shRNA (Thermo Fisher Scientific, Rockford, IL, USA) was used to express GFP in hPMFs. $2 \times 10^{5}$ SUIT-2 in $200 \mu \mathrm{l}$ PBS and $1 \times 10^{6}$ GFP-labeled hPMFs in $200 \mu 1$ PBS were transplanted into mice as described above. Peritoneally disseminated nodes were analyzed by stereoscopic microscopy (Zeiss, SteREO Lumar, Gottingen, Germany).

Flow cytometry analysis. Cultured cells were harvested by exposure to trypsin/EDTA for $5 \mathrm{~min}$ at $37^{\circ} \mathrm{C}$, washed in $10 \% \mathrm{FBS} / \mathrm{DMEM}$, suspended in ice-cold $1 \% \mathrm{FBS} / \mathrm{PBS}$ solution and analyzed using a flow cytometer (EC800 Cell Analyzer, Sony) equipped with a laser that provided an excita- 


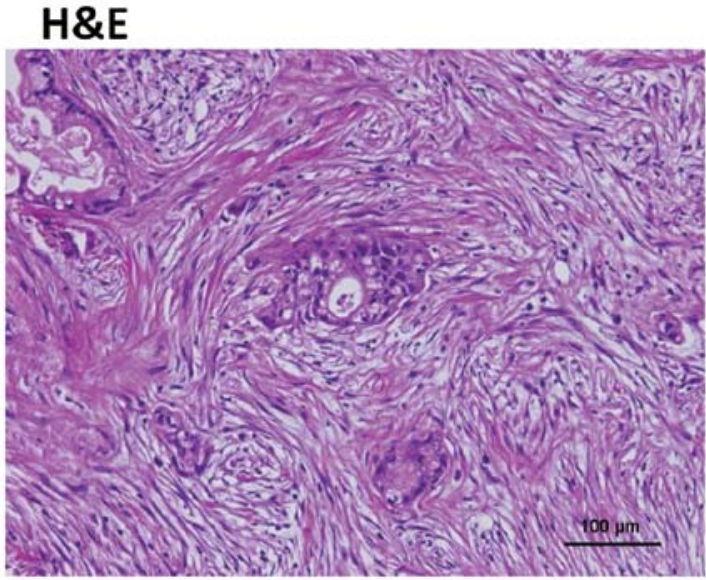

$\times 200$

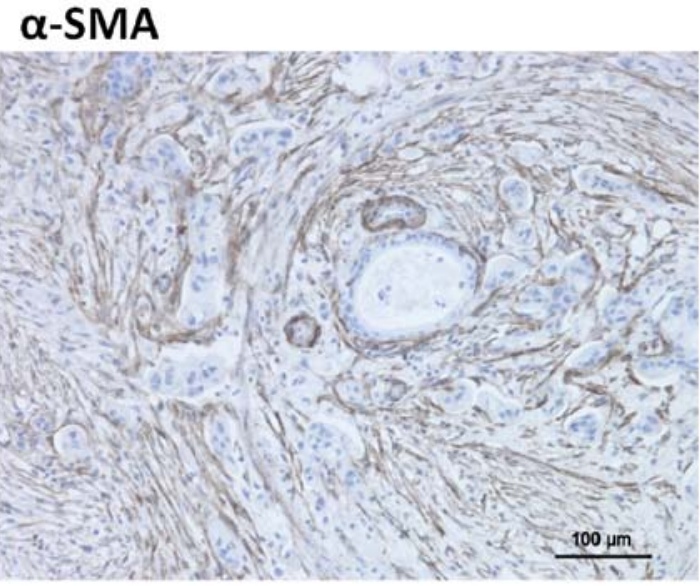

$\times 200$

Figure 1. Immunohistochemical analysis of $\alpha$-SMA expression at sites of peritoneal dissemination of human pancreatic cancer. The specimen was taken from a disseminated nodule of human pancreatic cancer. Many myofibroblasts were observed, as shown by H\&E staining (left panel) and were immunohistochemically positive for $\alpha$-SMA (right panel). Scale bars, $100 \mu \mathrm{m}$. Original magnification, $\mathrm{x} 200$.

A

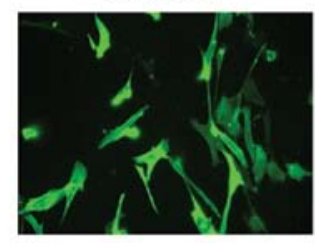

cytokeratin 18

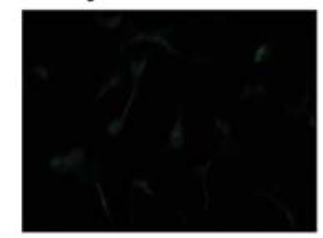

B

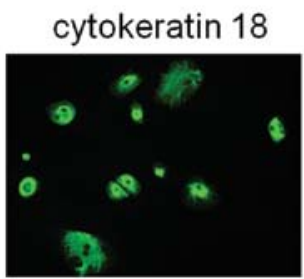

Vimentin

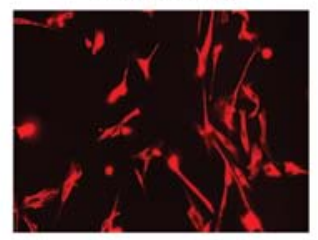

Vimentin

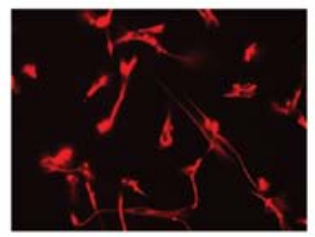

Vimentin

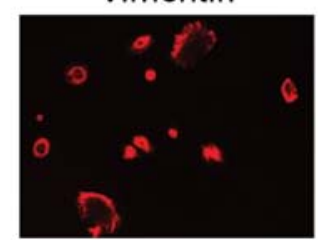

DAPI

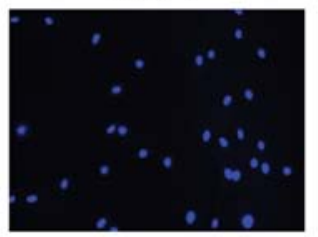

DAPI

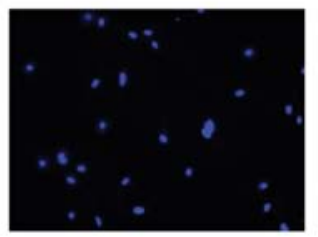

DAPI

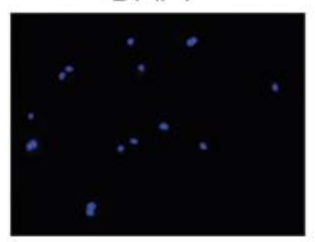

Merge

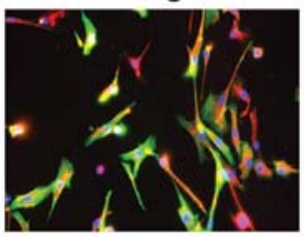

Merge
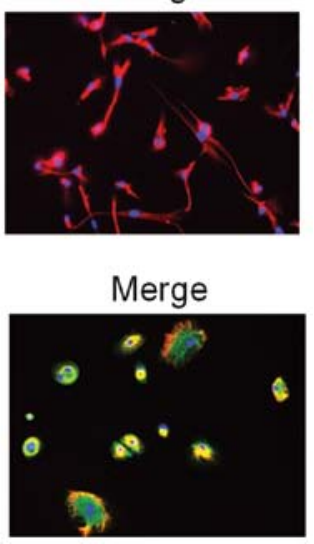

Figure 2. Immunofluorescent staining of $\alpha$-SMA, vimentin and cytokeratin 18 in hPMFs and human mesothelial cells. (A) All hPMFs expressed $\alpha$-SMA and vimentin but not cytokeratin 18. (B) Mesothelial cells expressed cytokeratin 18 and vimentin. Original magnification, x200.

tion wavelength of $488 \mathrm{~nm}$. Eclipse Analysis software (Sony) was used to quantify the fluorescent signals and set the logical electronic-gating parameters. Expression of GFP in original hPMFs and GFP-labeled hPMFs was analyzed by flow cytometry.

Statistical analysis. For in vitro and in vivo experiments, all values were expressed as mean $\pm \mathrm{SD}$ and compared using Student's t-tests. All experiments were performed at least three times. Statistical significance was defined as $\mathrm{P}<0.05$. All statistical analyses were performed using JMP 9 software (SAS Institute, Cary, NC, USA).

\section{Results}

Activated myofibroblasts are abundant in human peritoneally disseminated nodules. Specimens were obtained from peritoneally disseminated nodules of human pancreatic cancers. These nodules contained many activated hPMFs, as shown by their morphology (spindle-shaped cells; Fig. 1, left panel) and their positivity for $\alpha$-SMA staining (Fig. 1, right panel). Primary cultures of these hPMFs were positive for $\alpha$-SMA and vimentin and negative for CK18, suggesting that these cells were activated and were not contaminated by mesothelial cells (Fig. 2). 


\section{A}

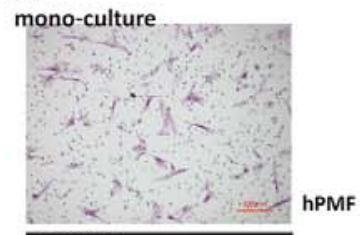

co-culture
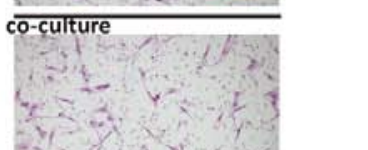

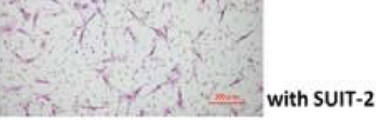

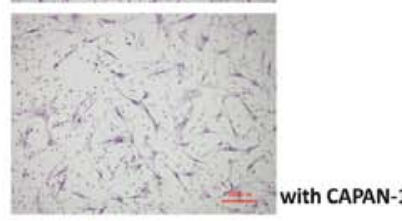

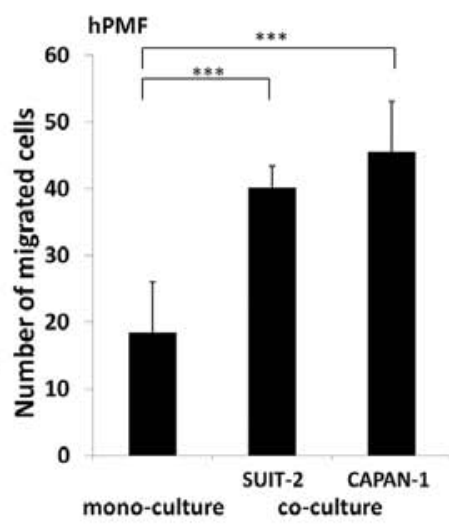

mono-culture co-culture

\section{B}

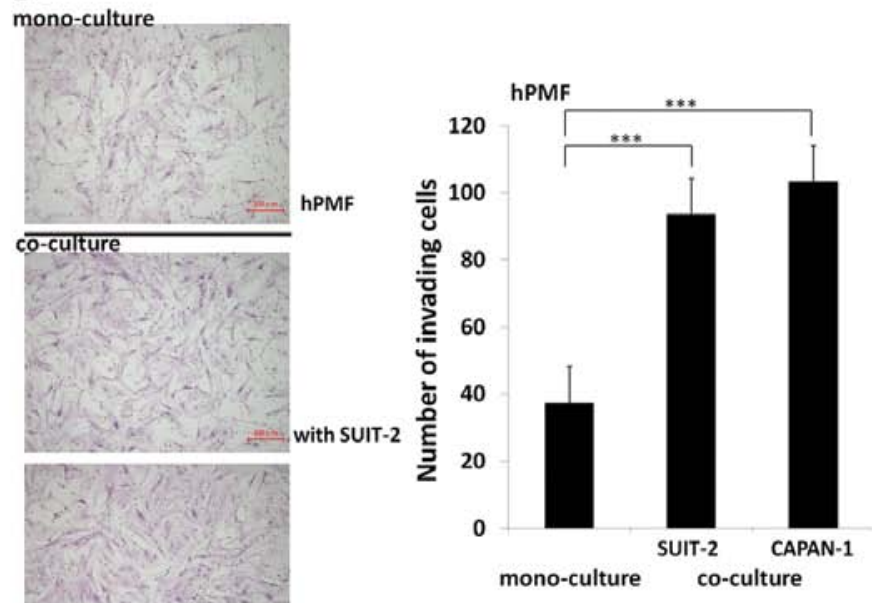

Figure 3. Mobility and invasiveness of hPMFs are enhanced when co-cultured with pancreatic cancer cells. (A) Representative microphotographs of the migration assay (left panel; H\&E staining, original magnification, x100). Co-culture with SUIT-2 or CAPAN-1 cells markedly stimulate the mobility of hPMFs (right panel,

$\left.{ }^{* * * *} \mathrm{P}<0.001\right)$. (B) Co-culture also markedly stimulated the invasiveness of hPMFs $\left({ }^{* * *} \mathrm{P}<0.001\right)$. H\&E staining; original magnification, $\mathrm{x} 100$.

A

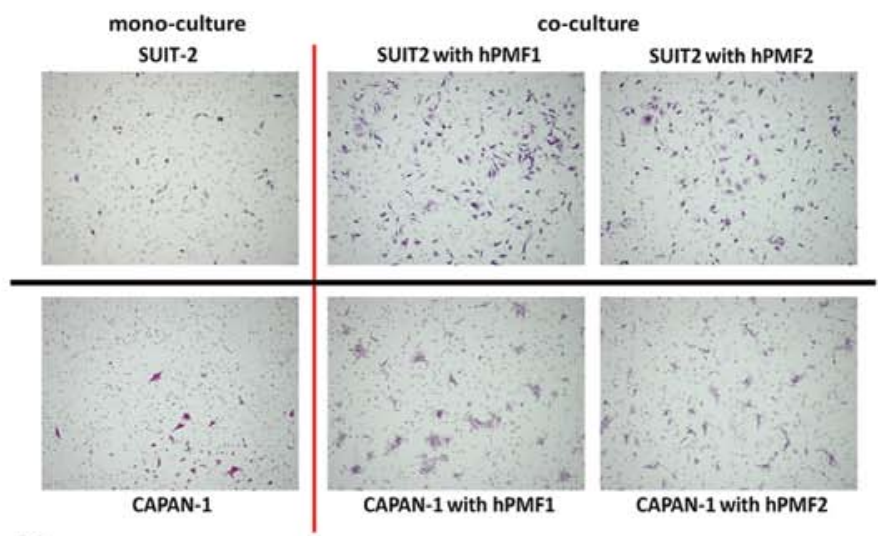

B

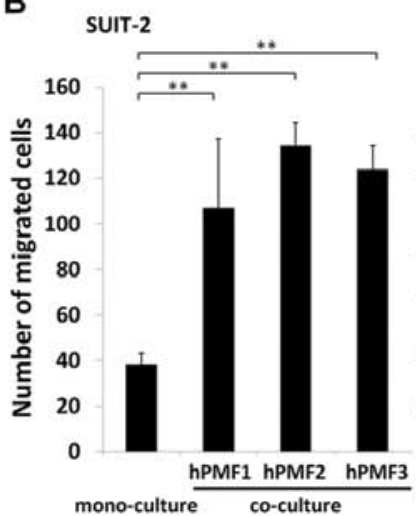

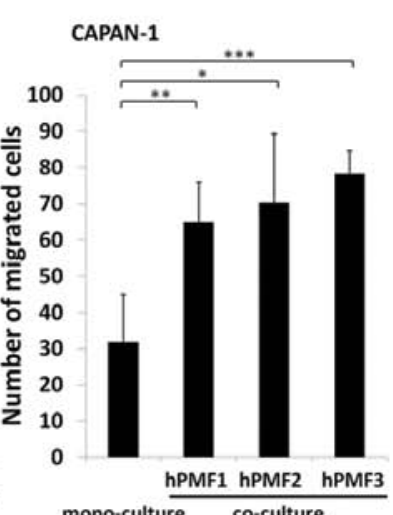

mono-culture co-culture
C

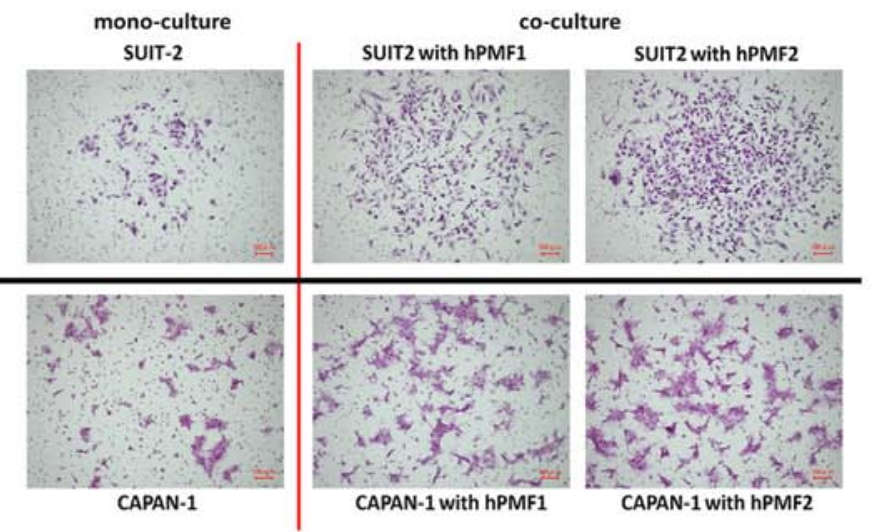

D
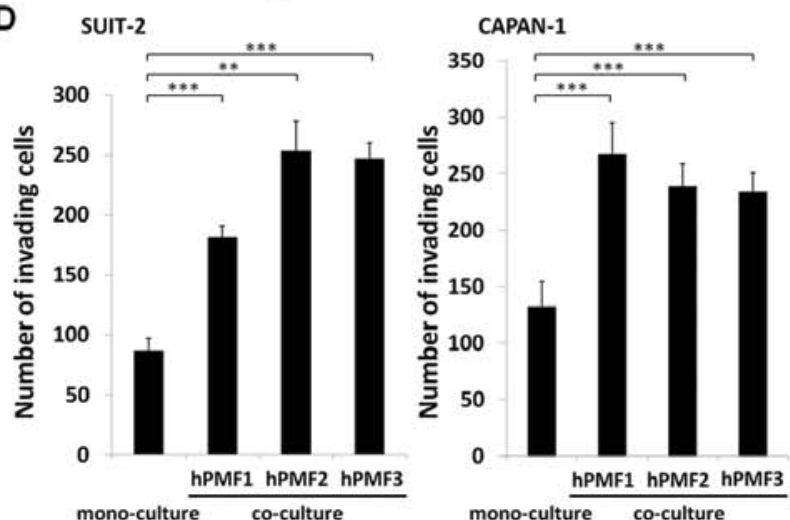

Figure 4. Mobility and invasiveness of pancreatic cancer cells are enhanced when co-cultured with hPMFs. (A and B) Representative microphotographs of the migration assay (H\&E staining, original magnification, $\mathrm{x} 100)$. Co-culture with hPMFs significantly stimulated the mobility of SUIT-2 and CAPAN-1 cells. ( $\mathrm{P}<0.05$, $\left.{ }^{* *} \mathrm{P}<0.01,{ }^{* * *} \mathrm{P}<0.001\right)$. (C and D) Co-culture also significantly increased the invasiveness of SUIT-2 and CAPN-1 cells $\left(^{* *} \mathrm{P}<0.01,{ }^{* * *} \mathrm{P}<0.001\right)$. H\&E staining; original magnification, $\mathrm{x} 100$.

Mobility and invasiveness of hPMFs are enhanced when co-cultured with pancreatic cancer cells. To investigate the cancer-stromal cell interactions between hPMFs and pancreatic cancer cells, we evaluated the effect of pancreatic cancer cells on hPMFs migration and invasiveness using indirect co-cultures. We found that both SUIT-2 and CAPAN-1 pancreatic cancer cells markedly stimulated the migration $(\mathrm{P}<0.001)$ (Fig. 3A) and invasiveness $(\mathrm{P}<0.001)($ Fig. 3B) of hPMFs. 
A

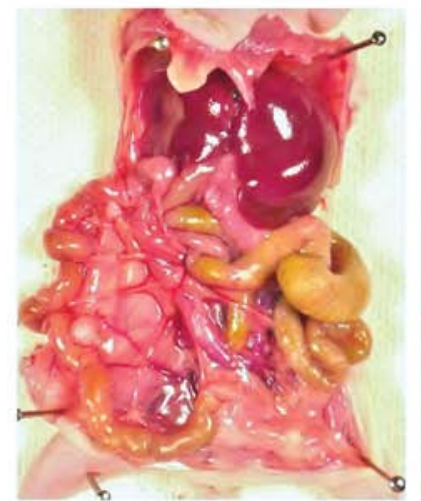

SUIT-2 + PBS

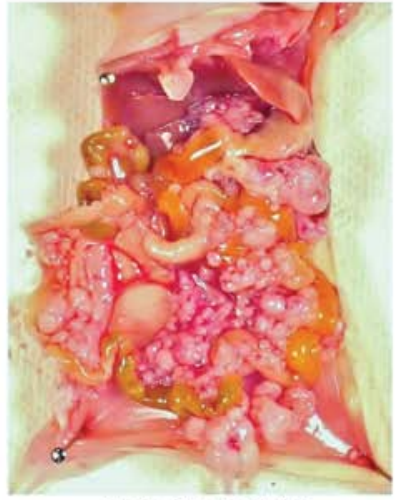

SUIT-2 + hPMF
B

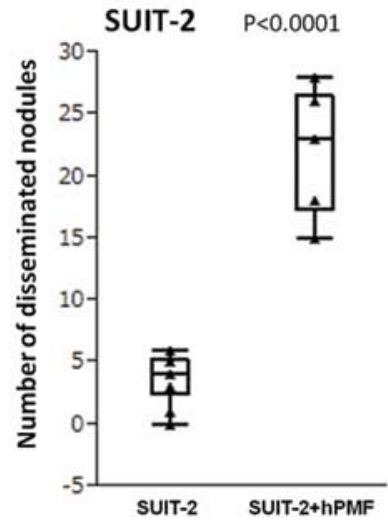

CAPAN-1 P $\quad P .0001$

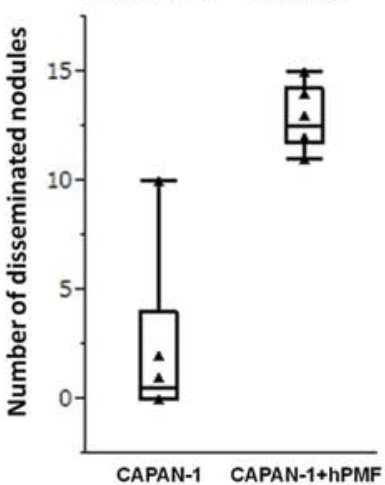

Figure 5. hPMFs promote the dissemination of pancreatic cancer cells in vivo. SUIT-2 and CAPN-1 cells, in the presence or absence of hPMFs, were intraperitoneally injected into BALB/c nu/nu mice. The mice were sacrificed after 28 days and disseminated nodules $>3 \mathrm{~mm}$ were counted. (A) Representative photographs of peritoneal dissemination in mice. PBS (left panel) or hPMFs (right panel, $1 \times 10^{6} /$ mouse) was intraperitoneally injected, together with $2 \times 10^{5}$ SUIT-2 or $1 \times 10^{6}$ CAPAN-1 cells. (B) Numbers of disseminated nodules were significantly greater in mice injected with cancer cells plus hPMFs than in mice injected with cancer cells alone (SUIT-2; $\mathrm{P}<0.0001$, CAPAN-1; $\mathrm{P}<0.0001$ ).

\section{$H \& E$}

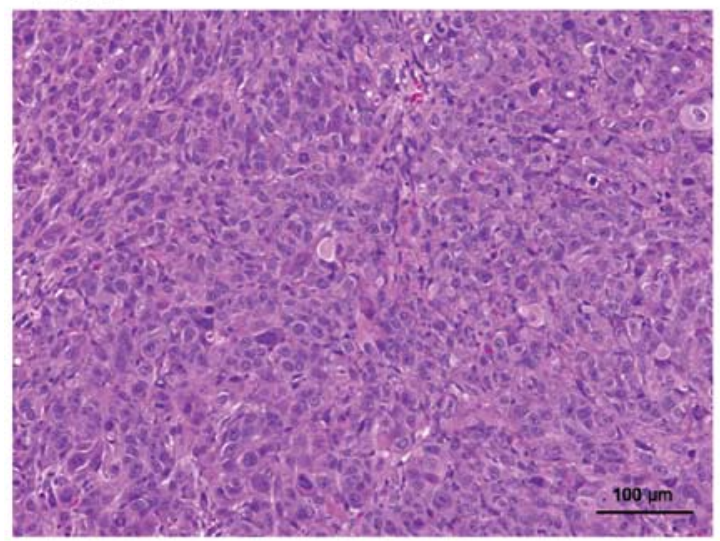

$\times 200$

\section{$\alpha-S M A$}

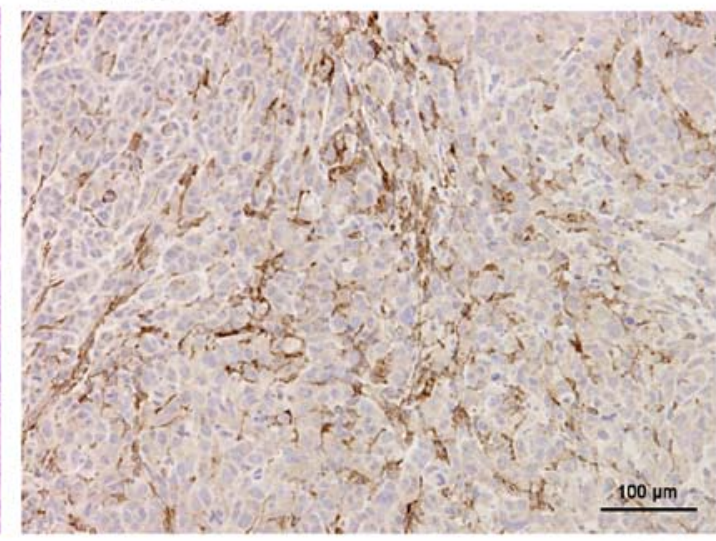

$\times 200$

Figure 6. Immunohistochemical analysis of $\alpha$-SMA in peritoneally disseminated nodules in mice. Specimens from disseminated nodules of mice intraperitoneally implanted with SUIT-2 and hPMFs contained a large number of activated myofibroblasts, as shown by H\&E staining (left panel) and by immunohistochemical staining for $\alpha$-SMA (right panel). Scale bars, $100 \mu \mathrm{m}$. Original magnification, $\mathrm{x} 200$.

Mobility and invasiveness of pancreatic cancer cells are enhanced when co-cultured with hPMFs. Similarly, we investigated the effect of co-cultured hPMFs on the mobility and invasiveness of pancreatic cancer cells. We found that hPMFs markedly stimulated the migration $(\mathrm{P}<0.05)$ (Fig. 4A and $\mathrm{B}$ ) and invasiveness $(\mathrm{P}<0.01)$ (Fig. $4 \mathrm{C}$ and D) of SUIT-2 and CAPAN-1 cells.

hPMFs promote the peritoneal dissemination of pancreatic cancer cells in vivo. Intraperitoneal implantation of cells into $\mathrm{BALB} / \mathrm{c}$ nu/nu mice, aged 5-6 weeks, was used to compare the dissemination over 28 days of pancreatic cancer cells alone and with hPMFs. Intraperitoneal injection of $2 \times 10^{5}$ SUIT- 2 cells plus $1 \times 10^{6}$ hPMFs (Fig. 5A, right panel) yielded a mean 22.5 \pm 4.9 peritoneally disseminated nodules $>3 \mathrm{~mm}$, compared with $3.8 \pm 2.0$ nodules in mice injected with SUIT-2 cells plus PBS (Fig. 5A, left panel), a difference that was statistically significant
$(\mathrm{P}<0.0001)$ (Fig. 5B, left panel). Similarly, injection of $1 \times 10^{6}$ CAPAN-1 cells plus $1 \times 10^{6} \mathrm{hPMFs}$ yielded a mean $12.8 \pm 1.5$ peritoneally disseminated nodules, compared with $2.5 \pm 4.0$ nodules in mice injected with CAPAN-1 cells plus PBS $(\mathrm{P}<0.0001)$ (Fig. 5B, right panel). H\&E staining and immunohistochemical analyses of nodules in mice injected with SUIT-2 cells plus hPMFs showed the presence of $\alpha$-SMA positive myofibroblasts (Fig. 6), similar to those observed human peritoneally disseminated nodules (Fig. 1).

hPMFs survive in peritoneally disseminated nodules of nude mice only when implanted together with pancreatic cancer cells. To investigate whether the hPMFs survive and exist in the peritoneally disseminated nodules after intraperitoneal implantation with or without pancreatic cancer cells, we used GFP-labeled hPMFs. Using flow cytometry, we evaluated the time-dependent changes in GFP expression of cultured hPMFs 

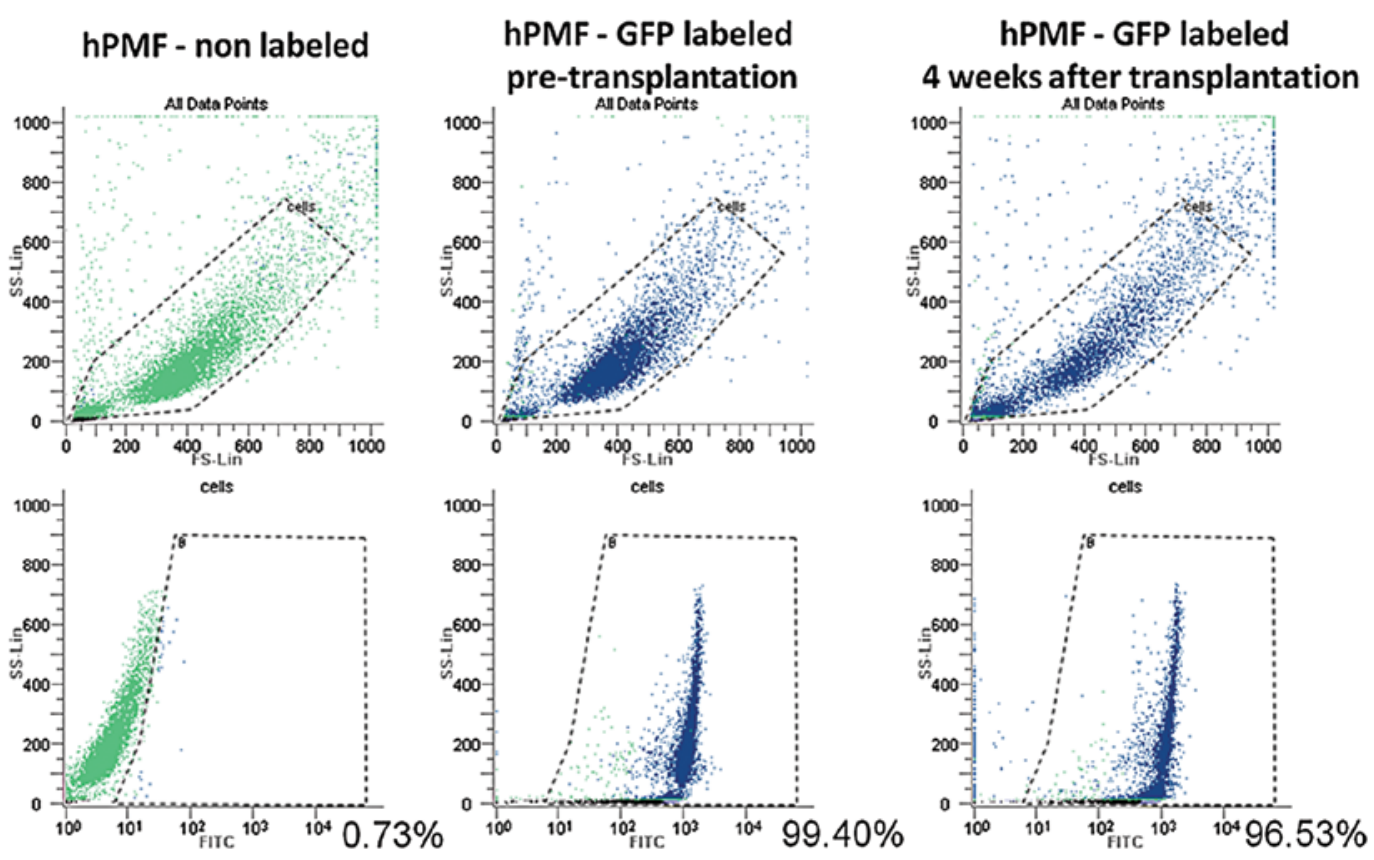

Figure 7. GFP expression of hPMFs in vitro. GFP expression of hPMFs was analyzed by flow cytometry. Primary cultures of original hPMFs and GFP-labeled hPMFs were analyzed on day 0 and day 28 after labeling. GFP expression did not decrease over 28 days.

A
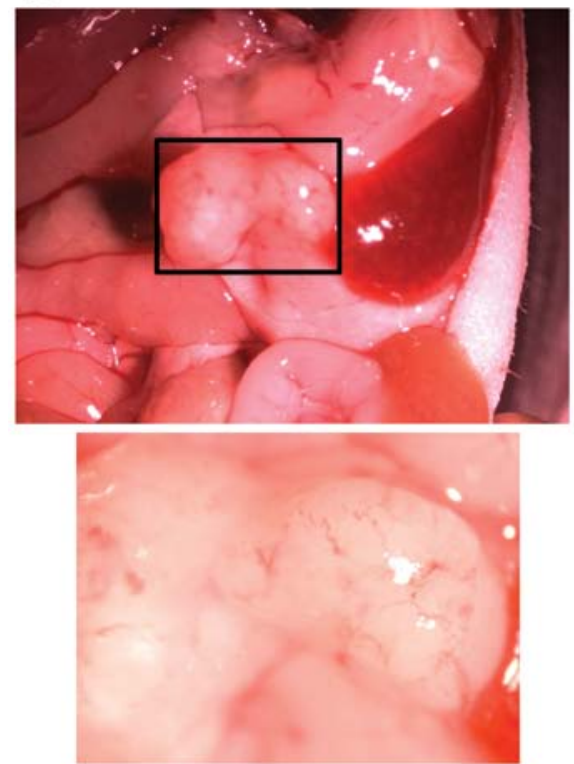

B
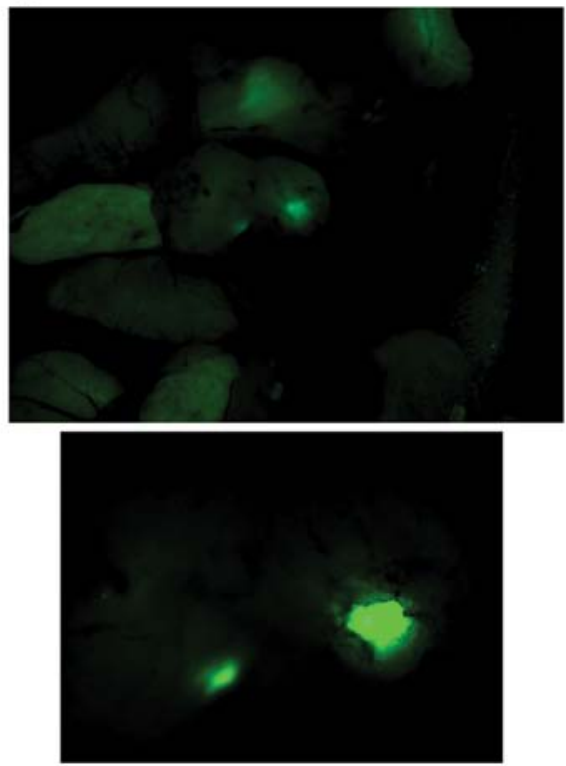

Figure 8. GFP-expressing hPMFs are presented in disseminated nodules in a mouse model. SUIT-2 cells $\left(2 \times 10^{5}\right)$ and GFP-labeled hPMFs $\left(1 \times 10^{6}\right)$ were intraperitoneally transplanted into mice. (A) A representative photograph of peritoneal dissemination in mice. The lower panel shows a magnification of the black square of the upper panel. (B) Corresponding fluorescence images observed by stereoscopic microscopy. GFP-labeled hPMFs were present in the disseminated nodules in nude mice 28 days after transplantation.

after labeling, finding that the expression of GFP in cultured hPMFs remained constant for 28 days after labeling (Fig. 7).

Intraperitoneal transplantation of $2 \times 10^{5}$ SUIT- 2 cells and $1 \times 10^{6}$ GFP-labeled hPMFs resulted in peritoneal dissemination in mice (Fig. 8A). Moreover, GFP-labeled hPMFs were observed in these peritoneally disseminated nodules 28 days after transplantation with pancreatic cancer cells (Fig. 8B).

\section{Discussion}

In the present study, we successfully isolated hPMFs by outgrowth from peritoneally disseminated nodules of pancreatic cancer and investigated the interaction between these primary cultured hPMFs and pancreatic cancer cells. We found that myofibroblasts were abundant around cancer cells at peritone- 
ally disseminated sites, similar to primary sites of pancreatic cancer, and that hPMFs promoted the migration and invasiveness of pancreatic cancer cells. Conversely, pancreatic cancer cells also promoted the migration and invasiveness of hPMFs. Our in vivo results revealed that number of peritoneally disseminated nodules was significantly greater when cancer cells were transplanted with hPMFs than when cancer cells were transplanted alone. In vivo experiments using GFP-labeled hPMFs showed that peritoneally disseminated nodules contained transplanted human myofibroblasts. Others also reported that myofibroblasts from human omentum were shown to promote the peritoneal dissemination of ovarian cancer cells in nude mice (14), and peritoneal myofibroblasts promoted the mobility and invasiveness of gastric cancer cells $(13,32)$. These findings suggest that hPMFs accelerate the malignant behavior of cancer cells through cancer-stromal cell interactions during the formation of peritoneally disseminated nodules.

Although several reports exist on methods of isolation of peritoneal myofibroblasts or mesothelial cells $(14,28)$, there is no difference between the two methods with the exception of the digestion time by trypsin/EDTA. These reports suggested that primary cultures of human peritoneal myofibroblasts and mesothelial cells could be contaminated with other cell types. In contrast, we established hPMFs from peritoneally disseminated nodules using an outgrow th method, similar to the method used to isolate human pancreatic stellate cells (hPSCs) from primary pancreatic cancers $(26,27)$. Microscopic observation of the primary cultured cells and in vitro immunofluorescence staining revealed that the isolated cells were spindle-shaped, positive for $\alpha$-SMA and vimentin, and negative for CK18, indicating that these isolated cells are activated hPMFs and that there was no contamination with hPMCs (28).

To confirm whether hPMFs, which were co-implanted with cancer cells, were present in the peritoneally disseminated nodules in mice, we used GFP-labeled hPMFs and found GFP positive cells in some disseminated nodules. Similar results were obtained using GFP-labeled hPSCs derived from primary pancreatic cancers. hPSCs transplanted into mouse pancreas with pancreatic cancer cells were shown to be present at peritoneally disseminated sites of pancreatic cancer (19). In addition, evaluation of the disseminated nodules derived from cancer cells co-transplanted with hPSCs in the peritoneal cavity showed that these hPSCs promoted the dissemination of pancreatic cancer cells in vivo, with no significant differences between hPMFs and hPSCs (data not shown). We also found that both hPMFs and hPSCs were not engrafted in the peritoneal cavity of nude mice if transplanted without cancer cells (data not shown). These results suggest that myofibroblasts liberated from their original tissues could engraft into the peritoneal cavities of mice when transplanted along with pancreatic cancer cells.

The peritoneum consists of a monolayer of mesothelial cells supported by a basement membrane that rests on a layer of connective tissue (24). Transforming growth factor- $\beta 1$ (TGF- $\beta 1$ ) derived from cancer cells in the peritoneal microenvironment was shown to activate hPMCs and transform these cells to myofibroblast-like cells (17). These findings focused on the role of hPMCs in the early phases of formation of peritoneally disseminated nodules of cancer cells. We found that hPMFs were abundant around cancer cells at peritoneally disseminated sites of pancreatic cancer, but we could not distinguish hPMC-derived myofibroblasts from other myofibroblasts.

hPMFs may derive from normal fibroblasts in the sub-mesothelial layer of the abdominal wall or from the transformation of mesothelial cells by TGF- $\beta 1$ or HGF $(16,17,25,33)$. Alternatively, hPMFs may be hPSCs liberated from the pancreas (19) or mesenchymal stem cells from the bone marrow (10). Further efforts are needed to identify the origin of these cells and the mechanism inducing their activation. These findings may contribute to the identification of new therapeutic targets to prevent the peritoneal dissemination of pancreatic cancer.

In conclusion, hPMFs can be isolated as primary cultures without contamination by hPMCs from disseminated nodules of pancreatic cancer. The cancer-stromal cell interactions between pancreatic cancer cells and hPMFs are important in the peritoneal dissemination of pancreatic cancer cells. Therapy targeting this interaction may improve the prognosis of patients with pancreatic cancer.

\section{Acknowledgements}

This study was supported in part by a Grant-in-Aid from the Ministry of Education, Culture, Sports, Science and Technology of Japan. Grant nos. 24390318, 25670586, 24390319, 25670585, 23390327, 25670584, 25293285 and 25670582.

\section{References}

1. Vincent A, Herman J, Schulick R, Hruban RH and Goggins M: Pancreatic cancer. Lancet 378: 607-620, 2011.

2. Sener SF, Fremgen A, Menck HR and Winchester DP: Pancreatic cancer: A report of treatment and survival trends for 100,313 patients diagnosed from 1985-1995, using the National Cancer Database. J Am Coll Surg 189: 1-7, 1999.

3. Chu DZ, Lang NP, Thompson C, Osteen PK and Westbrook KC: Peritoneal carcinomatosis in nongynecologic malignancy. A prospective study of prognostic factors. Cancer 63: 364-367, 1989.

4. Sadeghi B, Arvieux C, Glehen O, et al: Peritoneal carcinomatosis from non-gynecologic malignancies: results of the EVOCAPE 1 multicentric prospective study. Cancer 88: 358-363, 2000.

5. Armstrong T, Packham G, Murphy LB, et al: Type I collagen promotes the malignant phenotype of pancreatic ductal adenocarcinoma. Clin Cancer Res 10: 7427-7437, 2004.

6. Mahadevan D and Von Hoff DD: Tumor-stroma interactions in pancreatic ductal adenocarcinoma. Mol Cancer Ther 6: 1186-1197, 2007.

7. Feig C, Gopinathan A, Neesse A, Chan DS, Cook N and Tuveson DA: The pancreas cancer microenvironment. Clin Cancer Res 18: 4266-4276, 2012.

8. Hwang RF, Moore T, Arumugam T, et al: Cancer-associated stromal fibroblasts promote pancreatic tumor progression. Cancer Res 68: 918-926, 2008.

9. Apte MV, Park S, Phillips PA, et al: Desmoplastic reaction in pancreatic cancer: role of pancreatic stellate cells. Pancreas 29: 179-187, 2004.

10. Joyce JA and Pollard JW: Microenvironmental regulation of metastasis. Nat Rev Cancer 9: 239-252, 2009.

11. Strell C, Rundqvist H and Ostman A: Fibroblasts - a key host cell type in tumor initiation, progression, and metastasis. Ups J Med Sci 117: 187-195, 2012.

12. Fujita H, Ohuchida K, Mizumoto K, et al: Tumor-stromal interactions with direct cell contacts enhance proliferation of human pancreatic carcinoma cells. Cancer Sci 100: 2309-2317, 2009.

13. Yashiro M, Chung YS, Nishimura S, Inoue T and Sowa M: Fibrosis in the peritoneum induced by scirrhous gastric cancer cells may act as 'soil' for peritoneal dissemination. Cancer 77: 1668-1675, 1996. 
14. Cai J, Tang H, Xu L, et al: Fibroblasts in omentum activated by tumor cells promote ovarian cancer growth, adhesion and invasiveness. Carcinogenesis 33: 20-29, 2012.

15. Peron JM, Bureau C, Gourdy P, et al: Treatment of experimental murine pancreatic peritoneal carcinomatosis with fibroblasts genetically modified to express IL12: a role for peritoneal innate immunity. Gut 56: 107-114, 2007.

16. Yanez-Mo M, Lara-Pezzi E, Selgas R, et al: Peritoneal dialysis and epithelial-to-mesenchymal transition of mesothelial cells. N Engl J Med 348: 403-413, 2003.

17. Tsukada T, Fushida S, Harada S, et al: The role of human peritoneal mesothelial cells in the fibrosis and progression of gastric cancer. Int J Oncol 41: 476-482, 2012.

18. Epperly MW, Guo H, Gretton JE and Greenberger JS: Bone marrow origin of myofibroblasts in irradiation pulmonary fibrosis. Am J Respir Cell Mol Biol 29: 213-224, 2003.

19. Xu Z, Vonlaufen A, Phillips PA, et al: Role of pancreatic stellate cells in pancreatic cancer metastasis. Am J Pathol 177: 2585-2596, 2010.

20. Krist LF, Kerremans M, Broekhuis-Fluitsma DM, Eestermans IL, Meyer S and Beelen RH: Milky spots in the greater omentum are predominant sites of local tumour cell proliferation and accumulation in the peritoneal cavity. Cancer Immunol Immunother 47: 205-212, 1998.

21. Mochizuki Y, Nakanishi H, Kodera Y, et al: TNF-alpha promotes progression of peritoneal metastasis as demonstrated using a green fluorescence protein (GFP)-tagged human gastric cancer cell line. Clin Exp Metastasis 21: 39-47, 2004.

22. Sorensen EW, Gerber SA, Sedlacek AL, Rybalko VY Chan WM and Lord EM: Omental immune aggregates and tumor metastasis within the peritoneal cavity. Immunol Res 45: 185-194, 2009.

23. Tsujimoto H, Takhashi T, Hagiwara A, et al: Site-specific implantation in the milky spots of malignant cells in peritoneal dissemination: immunohistochemical observation in mice inoculated intraperitoneally with bromodeoxyuridine-labelled cells. Br J Cancer 71: 468-472, 1995.
24. Sugarbaker PH: Peritoneum as the first-line of defense in carcinomatosis. J Surg Oncol 95: 93-96, 2007.

25. Yashiro M, Chung YS, Inoue T, et al: Hepatocyte growth factor (HGF) produced by peritoneal fibroblasts may affect mesothelial cell morphology and promote peritoneal dissemination. Int J Cancer 67: 289-293, 1996.

26. Bachem MG, Schneider E, Gross H, et al: Identification, culture, and characterization of pancreatic stellate cells in rats and humans. Gastroenterology 115: 421-432, 1998.

27. Bachem MG, Schunemann M, Ramadani M, et al: Pancreatic carcinoma cells induce fibrosis by stimulating proliferation and matrix synthesis of stellate cells. Gastroenterology 128: 907-921, 2005

28. Jorres A, Ludat K, Lang J, et al: Establishment and functional characterization of human peritoneal fibroblasts in culture: regulation of interleukin- 6 production by proinflammatory cytokines. J Am Soc Nephrol 7: 2192-2201, 1996.

29. Ohuchida K, Mizumoto K, Murakami M, et al: Radiation to stromal fibroblasts increases invasiveness of pancreatic cancer cells through tumor-stromal interactions. Cancer Res 64: 3215-3222, 2004.

30. Ikenaga N, Ohuchida $\mathrm{K}$, Mizumoto $\mathrm{K}$, et al: $\mathrm{CD} 10^{+}$pancreatic stellate cells enhance the progression of pancreatic cancer. Gastroenterology 139: 1041-1051, 2010.

31. Sato N, Maehara N, Mizumoto K, et al: Telomerase activity of cultured human pancreatic carcinoma cell lines correlates with their potential for migration and invasion. Cancer 91: 496-504, 2001.

32. Yashiro M, Chung YS, Nishimura S, Inoue $\mathrm{T}$ and Sowa $\mathrm{M}$ : Peritoneal metastatic model for human scirrhous gastric carcinoma in nude mice. Clin Exp Metastasis 14: 43-54, 1996.

33. Lv ZD, Na D, Ma XY, Zhao C, Zhao WJ and Xu HM: Human peritoneal mesothelial cell transformation into myofibroblasts in response to TGF- $\beta 1$ in vitro. Int $\mathrm{J}$ Mol Med 27: 187-193, 2011 